\title{
Nuevas formas de aprender: La formación docente frente al uso de las TIC
}

\section{New Ways of Learning: Teacher Training in the Use of ICTs}

\author{
Ronald M. Hernández* Universidad San Ignacio de Loyola, Lima, Perú. \\ Universidad de San Martín de Porres, Lima Perú. \\ ORCID: https://orcid.org/0000-0003-1263-2454 \\ Rosalina Orrego Cumpa Universidad César Vallejo, Lima, Perú. \\ ORCID: https://orcid.org/0000-0001-9914-9195
}

Sonia Quiñones Rodríguez Universidad Seminario Evangélico de Lima, Lima, Perú. ORCID: https://orcid.org/0000-0003-4554-7176

Recibido 15-06-18 Revisado 30-06-18 Aprobado 27-09-18 En línea 05-10-18

\section{*Correspondencia}

Email: rhernanderzv@usil.edu.pe

\section{Citar como:}

Hernández, R.M., Orrego, R., \&Quiñones, S. (2018 Nuevas formas de aprender: La formación docente en el uso de las TIC, Propósitos y Representaciones 6(2), 671-701. Doi: http:// dx.doi.org/10.20511/pyr2018.v6n2.248

(C) Universidad San Ignacio de Loyola, Vicerrectorado de Investigación, 2018

(cc) BY-NC-ND Este artículo se distribuye bajo licencia CC BY-NC-ND 4.0 Internacional (http://creativecommons.org/licenses/by-nc-nd/4.0/). 


\section{Resumen}

Las tecnologías de la información y comunicación (TIC), se han convertido en un recurso determinante en el campo educativo, y a la vez una variable indispensable en la práctica académica donde se busca aprovechar cada uno de los recursos que nos ofrece la Web 2.0. El objetivo de este artículo es realizar un análisis y revisión crítica de los aspectos conceptuales frente a la formación del docente, en el uso de las TIC y su implicancia en su labor diaria, así como en el proceso de enseñanza-aprendizaje. Se concluye en resaltar la importancia de la creación de dimensiones pedagógicas que señalen las competencias de formación, que debe presentar un docente, frente a la nueva tendencia tecnológica.

Palabras clave: Formación docente; TIC; aprendizaje; conocimiento pedagógico. 


\section{Summary}

Information and communication technologies (ICTs) have become a decisive resource in the field of education, and at the same time, an indispensable variable in the academic practice where we seek to use each one of the resources offered by Web 2.0. The objective of this article is to perform a critical analysis and review of the conceptual aspects of teacher training, the use of the ICTs and their implications in teacher's daily work, as well as in the teaching-learning process. Finally, it is concluded to highlight the importance of the creation of pedagogical dimensions that indicate the training competencies a teacher must have given the new technological tendency.

Keywords: Teacher training; ICT; learning; pedagogical knowledge 


\section{Introducción}

Las Tecnologías de la Información y Comunicación (TIC) remontan su origen a la aparición de las computadoras y el internet, impulso, que conllevo a la facilidad del acceso de información, dando lugar a la creciente e importancia sociedad tecnológica. Siendo así, desde un punto histórico, la revolución tecnológica ha marcado un decisivo "quiebre" en la sociedad mundial, y su repercusión en la vida humana, suponiendo un salto cualitativo en la forma en que el hombre encuentra sus relaciones con sus pares y la redefinición de la adquisición del nuevo conocimiento, entendiendo así, a las TIC como agente externo, generador de conocimiento; redefiniendo los modelos de relación, tratamiento y comunicación de la información.

Actualmente el uso de las TIC está ampliamente extendido ocasionando cambios en las últimas décadas en diferentes aspectos de la vida humana, para Jacovkis (2012) las TIC han causado un profundo impacto en todo el mundo en la ciencia y en la tecnología y por consiguiente en la sociedad. La UNESCO (1998) menciona que el rápido progreso de las TIC modifica la forma de elaboración, adquisición y transmisión de conocimientos. Estos cambios y modificaciones a las cuales se hace referencia repercuten la economía, sociedad, política y cultura; convirtiendo al ser humano en un ser cada vez más creativo, crítico, autónomo, en la búsqueda de adquirir nuevas competencias para su adecuado desenvolvimiento en un contexto que cada día es más variable e incierto; así para Cornella (2000) las sociedades del conocimiento son las organizaciones y las personas que se enfrentan a la necesidad de gestionar la información de manera eficiente.

Cabero (2007) menciona que las TIC, dentro de los nuevos contextos, son elementos esenciales en la interacción de los individuos; asimismo, Carneiro, Toscano y Díaz (sf) acompañan la concepción anterior indicando que el desarrollo acelerado de la sociedad de la información supone nuevos retos para la educación y aprendizaje. Estas dos últimas definiciones inician un nuevo desafío en el ámbito educativo, donde el conocimiento y uso de 
las TIC reconfiguran en el estudiante y todo aquel agente que lo rodea, la preparación, formación y adecuado uso, de éstas, como herramientas pedagógicas capaces de brindar en el estudiante un instrumento de acompañamiento educativo. Las nuevas tecnologías, generan metodologías diversas, generando cambios en el su uso didáctico y pedagógico, es en este ámbito, que se generan herramientas que posibilitarán el análisis, reflexión y estudio del binomio educación y tecnología (Cabero, 2007).

El uso de la tecnología en la educación, ha permitido que la flexibilidad y la capacidad de adaptación de los estudiantes logré ser una herramienta de interacción y de facilidad de uso en el aprendizaje; sin embargo, la forma en que la tecnología se ha aplicado a la educación, ha ido variando con cada avance permitiendo mayor eficiencia y aprovechamiento en los recursos educativos para la optimización del proceso enseñanza-aprendizaje. Fernández (2001) la presencia de las nuevas tecnologías exige una profunda reflexión en busca de mejoras educativas y su adaptación a la actividad educativa cotidiana. Así pues, estas nuevas tecnologías han ocasionado un cambio en la educación, tanto en los usuarios que participan de la misma y los escenarios donde ocurre el aprendizaje (Domínguez, 2004).

Es bastante claro que se están dando grandes pasos en relación con la incorporación de tecnologías en las instituciones educativas. Desde el siglo pasado se ha experimentado una fuerte revolución tecnológica, facilitando así el acceso a la información que se desea obtener, como consecuencia la convivencia entre el hombre y los nuevos aparatos tecnológicos, por ello se hace necesario poder estudiar esta simple o tan compleja interacción.

Desde esta perspectiva, a la educación como motor de desarrollo y cambio social, le corresponde formar un ser humano que responda a las características requeridas para la sociedad actual. Ante ello Escudero (1992) dice que es necesario integrar las nuevas tecnologías en un programa educativo bien fundamentado para hacer un uso pedagógico de las mismas, ya que son las metas, objetivos, contenidos y metodología lo que les permiten adquirir un sentido educativo. Con el avance de la tecnología y su creciente 
incorporación en la educación, han hecho que las aulas de clase comiencen a asumir un nuevo rol protagónico de transformación; como señala Coll, Mauri y Onrubia (2008) el impacto de las TIC en los procesos educativos ha aumentado progresivamente, en paralelo a la creciente incorporación de estas tecnologías en todos los niveles de enseñanza; así pues De la Torre y Domínguez, (2012) dicen que el Proceso de Enseñanza Aprendizaje (PEA) en nuestros días tiene como propósito contribuir a la formación del estudiante, a través del cumplimiento de objetivos instructivos y educativos. Kay y Knaack (2009) plantean que el PEA apoyado con las TIC, facilitan tanto la enseñanza como el aprendizaje, incluso en mayor medida que los medios tradicionales como son los libros y el televisor, entre otros.

La adaptación de la sociedad, en referencia a la educación, lleva al desarrollo de vías que puedan integrar las TIC en los procesos formativos. La educación superior concebida por Irigoyen, Jiménez y Acuña (2001), como aquella formación que parte de modelos basados en una concepción de los contenidos de aprendizaje está siendo modificada, debido al acelerado cambio de los conocimientos. La necesidad de adaptación en este contexto, repercute en la concepción de cambio, de estudiantes como usuarios y del rol docente; todo ello implica, a su vez, cambios en los cánones de enseñanzaaprendizaje hacia un modelo cada vez más flexible en el marco de un proceso de innovación (Salinas, 2004).

Es en este contexto donde el docente, desarrolla una serie de contingencias de reforzamiento y control de estímulos para enseñar, se habla entonces, de un manejo adecuado de los recursos tecnológicos, en mejora de adquirir buenos niveles de eficacia en su enseñanza y éxito en el aprendizaje de sus alumnos. (Guerreo \& Faro, 2012). Domínguez (2004) en la actualidad el interés por la producción de las TIC en el sistema educativo se ve reflejado por conocer la base formativa de los docentes y su implicancia en la adquisición del conocimiento.

Toda innovación o mejora educativa requiere que los docentes asuman un rol activo en su implementación. Estas innovaciones o mejoras educativas 
no siempre pueden incorporarse con facilidad, Marqués (2004) señala que las TIC se han convertido en un eje transversal de toda acción formativa y donde casi siempre tendrán una triple función: (1) como instrumento facilitador de los procesos de aprendizaje, (2) como herramienta para el proceso de la información y (3) como contenido implícito de aprendizaje. Izquierdo y Pardo (2007) mencionan que el empleo de las TIC en el proceso docente educativo y sobre todo en la educación superior ha evidenciado la necesidad de transformar el trabajo metodológico y la formación de los docentes y otros sujetos que participan en dicho proceso, para que puedan enfrentar los retos que en cuanto a la formación de los profesionales necesita la sociedad actual; esto quiere decir que el grado de utilización de las TIC influye en el impacto que estas pueden generar en el PEA. Concluyendo así que hoy en día los formadores necesitan utilizar las TIC en muchas de sus actividades profesionales habituales para mejorar el quehacer educativo, pero qué sucede cuando el docente no está persuadido o capacitado para llevar a cabo estas actividades. Distintas resistencias basadas en el desinterés, desinformación o prejuicios, pueden invalidar cualquier intento de mejora en la educación, es así que la concepción de un docente dirigiendo clases magistrales hacia un estudiante pasivo se han visto obligadas a ser repensadas, para centrarse en una formación más dirigida al alumno, donde éste tome un papel más activo, haciendo uso de medios interactivos, y se confluya hacia una relación más colaborativa de aprendizaje.

Es desde este punto, que se pretende realizar una revisión sistemática sobre las actitudes y el conocimiento pedagógico, como aquellas variables que influyen dentro del proceso de formación docente y su repercusión para el estudiante. Las actitudes como concepto reflejan la importancia del funcionamiento psicológico del ser humano, tanto en su inserción laboral como educativa. Ferreira, (2009) una actitud es una tendencia a la acción adquirida en el ambiente en que se vive y derivada de experiencias personales, es aquí donde aquello que propicia el comportamiento, llega a conformar parte de un sistema de representación de la realidad. Aunque el concepto de 
actitud es aún muy difícil de definir, pues como menciona Dawes (1975), es más fácil medir las actitudes, que definirlas. Es en definición de Hollander (2000) que se explica a las actitudes como percepciones acerca de las personas, las cosas o los hechos ambientales; asimismo, que en la medida en que dirigen la conducta, tienen cualidades motivacionales. Y explica que este concepto se asocia a la variable denominada "valores". Es en este contexto donde, las actitudes docentes buscan aportar a las prácticas educativas a nivel de formación y manejo de herramientas, así como el manejo didáctico de las mismas.

\section{Conocimiento pedagógico frente a las TIC.}

El conocimiento pedagógico está relacionado con el saber educar para un tipo de sociedad, es en este punto donde se describe tres aspectos de este tipo de conocimiento: teórico, práctico y crítico; el primero busca identificar los fenómenos educativos; el segundo, busca actuar para potenciar a la persona y a la sociedad; por último el tercero fundamenta la práctica educativa; también, es reflexión crítica sobre la práctica pedagógica. Touriñan, (1988) busca entender y dominar la educación a través de los componentes del fenómeno educativo que hay que entender para dominar dicho fenómeno, Los estudios sobre el conocimiento del docente se han convertido en un fructífero campo de investigación, la formación inicial y permanente del profesorado se analizan en función del conocimiento y enseñanza interactiva. (Schubert, Medina \& do Prado, 2011)

Si relacionamos lo anterior con el contexto educativo, el interés recae sobre la formación docente en el empleo y adaptabilidad a estas nuevas tecnologías y la repercusión que trae frente al PEA, es aquí donde el rol del docente adquiere suma importancia al considerarse un mediador de aprendizaje, respetando y valorando los nuevo desafíos frente a una educación en la era del conocimiento. 
Los procesos de enseñanza deben conducir a una seria reflexión del docente, no siempre llevada a cabo, sobre la importancia de tener conocimiento acerca de cuál es el uso adecuado que se le debe dar a las TIC para lograr una verdadera integración en la educación; es así que se debe dar un cambio frente a la concepción de su uso en relación al para qué y por qué utilizarlas.

Twining, (2002) en este contexto, y para tratar de comprender el impacto de las TIC en la educación, se ha planteado cada vez con más fuerza la necesidad de estudiar de manera empírica la manera en que profesores y alumnos usan estas nuevas tecnologías en el desarrollo real de las prácticas que llevan a cabo en el aula, considerando evaluar la manera como se viene realizando los procesos de capacitación docente frente a estos nuevos cambio y la actitud frente al asumir mayores responsabilidades.

Lo mencionado anteriormente ayuda a definir un perfil del docente, flexible y variante, capaz de adecuarse a los continuos cambios que acontecen en nuestra sociedad (Bozu \& Canto, 20009). De esta manera el rol del docente es variante hacia nuevas formas de relación con los estudiantes y su entorno de aprendizaje, los escenarios donde se lleva a cabo una sesión de clase adquiere un mayor protagonismo en base al uso de las nuevas tecnologías. (Llorente, Cabero \& Barroso, 2015).

La definición del conocimiento pedagógico se refiere a las actividades pedagógicas que un docente podría utilizar, los procesos y prácticas del método de enseñanza y cómo estos se relacionan con el pensamiento. (Cabero Almenara, Marín Díaz, \& Castaño Garrido, 2015). Su utilización permite la participación y la realización de tareas ajustadas a las necesidades e intereses del alumnado (Fernández Batanero, \& Rodríguez Martín, 2017).

Aunque las TIC puede considerarse una eficaz herramienta para mejorar la educación del alumnado, es necesario una transformación de las actuaciones docentes, ya que el aprendizaje se relaciona con la calidad de las prácticas en las que participan dentro del aula (Coll, Mauri, y Onrubia, 
2008). Durante los últimos años se ha mencionado sobre la problemática de la capacitación en TIC de los docentes y las competencias que necesitan para su utilización (Fernández-Batanero y Bermejo, 2012; Rosario y Vázquez, 2012; Ortiz, Almazán, Peñaherrera, y Cachón, 2014; Rangel, 2015). Uno de los retos en esta sociedad del conocimiento es incorporar la formación del docente, nuevas competencias que impliquen el uso de las TIC en la enseñanza y aprendizaje, integrando estos aspectos al logro de objetivos pedagógicos.

La formación del docente y la adquisición de los conocimientos para una buena práctica y manejo de las TIC en el aula, coinciden con lo que plantea Cabero, (2004; 2008; 2014):

Cuadro 1. Formación y adquisición de conocimientos frente a las TIC.

\begin{tabular}{|c|c|}
\hline $\begin{array}{l}\text { Formación/ } \\
\text { Conocimientos }\end{array}$ & Características \\
\hline Instrumental & Competencias mínimas para el manejo instrumental de las diferentes TIC \\
\hline Semiológica/Estética & Decodificación de mensajes que usan las TIC \\
\hline Curricular & Las TIC son un medio para el alcance de objetivos curriculares propuestos. \\
\hline Pragmática & $\begin{array}{l}\text { Las diferentes acciones que se proponen al hacer uso de las TIC, permiten } \\
\text { la obtención de una serie de productos sociocognitivos diferenciados. }\end{array}$ \\
\hline Psicológica & $\begin{array}{l}\text { Las TIC sí desarrollan habilidades cognitiva específicas, se convierten en } \\
\text { un recurso de enseñanza-aprendizaje. }\end{array}$ \\
\hline Productora/Diseñador: & $\begin{array}{l}\text { El docente debe convertirse en un productor de las TIC. El inicio se ve } \\
\text { marcado por el consumismo; sin embargo, debe alcanzarse el ideal de } \\
\text { producción. }\end{array}$ \\
\hline Selección/Evaluación: & $\begin{array}{l}\text { El docente debe convertirse en un curador de contenidos, propio de una } \\
\text { selección y evaluación de las TIC. }\end{array}$ \\
\hline Crítica & $\begin{array}{l}\text { Es un recurso realista, que lleva a la educación a alinearse a la perspectiva } \\
\text { de la nueva sociedad el conocimiento. }\end{array}$ \\
\hline \multirow[t]{2}{*}{ Organizativa } & $\begin{array}{l}\text { No solo existe un recurso, las TIC nos muestran una gama de estrategias } \\
\text { que permitirán que el docente organice los contenidos para una mejor } \\
\text { obtención de resultados. }\end{array}$ \\
\hline & $\begin{array}{l}\text { Las TIC se convierten en un recurso aliado para el docente, y la ceración de } \\
\text { nuevos espacios formativos, negando el rechazo y permanente sumisión. }\end{array}$ \\
\hline Investigadora & $\begin{array}{l}\text { No solamente se convierte en un recurso para obtener resultados, sino para } \\
\text { la obtención de los mismos. }\end{array}$ \\
\hline Comunicativa & $\begin{array}{l}\text { Se establecen diferentes modelos de comunicación sincrónica y } \\
\text { asincrónica, creando diferentes escenarios de comunicación con las TIC. }\end{array}$ \\
\hline
\end{tabular}




\section{Conclusiones}

La integración de las TIC en el campo educativo depende de diversas características, que en su conjunto favorecerán la metodología del docente. El conocimiento pedagógico es un valor determinante frente al proceso de enseñanza; sin embargo, el aspecto socioemocional (percepciones actitudes) que tengan los docentes respecto a estos medios de comunicación, serán factores determinantes a la hora de utilizarlos e integrarlos en los procesos educativos.

En la actualidad, el docente concibe el uso de las TIC como un reto y la actualización de estos procesos conlleva a mejorar la práctica educativa (Álvarez, et al. 2011). Este panorama permite revertir la educación tradicional y al docente permitirle adaptarse al cambio y convertirse en el agente predominante de estos nuevos recursos.

Integrar las TIC en el campo de la formación está pasando por un cambio sustancial, donde se concibe a estos recursos y la imagen del docente como un facilitador del aprendizaje, donde las TIC son un elemento de ayuda para dinamizar el proceso de aprendizaje (Marín \& Romero 2009), para ello se requiere de un conjunto de competencias que el docente debe adquirir con la lógica de sumar una metodología capaz de aprovechar las herramientas tecnológicas, donde la capacitación docente deberá considerarse una de las primeras opciones antes de afrontar nuevos retos educativos (Hernández, 2017).

Este nuevo reto de adquirir conocimientos pedagógicos relacionadas a las TIC, vivencia su reto en la formación universitaria del futuro docente, Marín y Romero (2009) indican que la comunicación, la creatividad y la gestión del cambio se han convertido en tres disciplinas que marcarán el devenir de la educación universitaria de hoy.

Estas nuevas propuestas de formación del docente en TIC y la adquisición de conocimientos pedagógicos frente al uso de estos recursos indica que el docente debe tener competencias en diferentes dimensiones, lo que permitirá 
el uso objetivo de las TIC y su posibilidad de adecuación a las características educativas que se desea impartir.

La formación del docente debe ser integral desde una perspectiva que integre a la tecnología como un recurso para la enseñanza y la obtención del aprendizaje del estudiante y ello lleva a la formación de un docente con un manejo reflexivo, constructivista y evaluador en la obtención de nuevos productos. No obstante, la expansión de las TIC en nuestra sociedad presenta una serie de limitaciones, como problemas técnicos, de seguridad y limitaciones económicas y culturales (Marín \& Romero, 2009) sin embargo, ello no debe ser limitante para que la formación del docente siga acrecentando la búsqueda de nuevas alternativas de aprendizaje con recursos tecnológicos.

\section{Referencias}

Álvarez, S., Cuéllar, C., López, B., Adrada, C., Anguiano, R., Bueno, A., \& Gómez, S. (2011). Actitudes de los profesores ante la integración de las TIC en la práctica docente: estudio de un grupo de la Universidad de Valladolid. Edutec. Revista electrónica de tecnología educativa, 35, Recuperado de: http://www.edutec.es/revista/index.php/edutec-e/article/ view/416/152

Bozu, Z., \& Canto, P. J. (2009). El profesorado universitario en la sociedad del conocimiento: competencias profesionales docente. Revista de Formación e Innovación Educativa Universitaria, 2(2), 87-97.

Cabero, J. (2004). Formación del profesorado en TIC. El gran caballo de batalla. Comunicación y Pedagogía, 195, 27-31.

Cabero, J. (2008). La formación del profesorado para el manejo de las TIC en los procesos de enseñanza/ aprendizaje. En F. Martínez (coord.). Incorporación de las TIC en los programas académicos de las Universidades Estatales Costarricenses. (pp. 55-68). Murcia, Diego Marín.

Cabero, J. (2007). Las necesidades de las TIC en el ámbito educativo: oportunidades, riesgos y necesidades. Tecnología y Comunicación Educativa, 21(45), 5-19. 
Cabero-Almenara, J., \& Díaz, V. M. (2014). Miradas sobre la formación del profesorado en tecnologías de información y comunicación (TIC). Enl@ce: Revista Venezolana de Información, Tecnología y Conocimiento, 11(2), 3.

Cabero Almenara, J., Marín Díaz, V., \& Castaño Garrido, C. (2015). Validación de la aplicación del modelo TPACK para la formación del profesorado en TIC. @tic. Revista d'innovació educativa, 14, 13-22. Doi: http://doi.org/10.7203/attic.14.4001

Carneiro, Toscano \& Díaz (sf). Los desafíos de las TIC para el cambio educativo. Madrid: Fundación Santillana.

Coll, C., Mauri Majos, M., \& Onrubia Goni, J. (2008). Análisis de los usos reales de las TIC en contextos educativos formales: una aproximación socio-cultural. REDIE, 10(1), 1-18. Recuperado de: https://redie.uabc. $\mathrm{mx} /$ redie/article/view/177/848

Cornella, A. (2000). La gestión de la información en la organización. Bilbao: Deusto.

Dawes, R. (1975). Fundamento y técnicas de medición de actitudes. México: Ed. Limusa

De la Torre, L., \& Dominguez, J. (2012). Las TIC en el proceso de enseñanza aprendizaje a través de los objetos de aprendizaje. RCIM, 4(1), 83-92.

Domínguez, R. (2004). Nuevas Tecnologías y Educación en el siglo XXI. Etic@net, 4. Recuperado de:http://www.ugr.es/ sevimeco/ revistaeticanet/Numero4/Articulos/Formateados/NTIC_SXXI.pdf

Escudero, J. M. (1992). La integración escolar de las nuevas tecnologías de la información. España: Infodidac 21

Fernandez M. (2001). La aplicación de las nuevas tecnologías en la educación. Tendencias Pedagógicas, 6, 139-148. Recuperado de: https://revistas. uam.es/tendenciaspedagogicas/article/view/1811

Fernández-Batanero, J.M., \& Bermejo, B. (2012). Actitudes docentes hacia las TIC en centros de buenas prácticas educativas con orientación inclusiva. Enseñanza \& Teaching, 30(1), 45- 46 doi: https://doi. org/10.30552/ejihpe.v7i3.204

Fernández Batanero, J. M., \& Rodríguez Martín, A. (2017). TIC y diversidad funcional: conocimiento del profesorado. EJIHPE. European Journal 
of Investigation in Health, Psychology and Education, 7(3), 157-175. Recuperado de: https://idus.us.es/xmlui/handle/11441/69546

Ferreira, M. (2009). Cambio de actitudes sociales para un cambio de vida. Recuperado de: http://www.um.es/discatif/documentos/Actitudes Cuenca09.pdf

Guerrero, F., \& Faro, T. (2012). Breve análisis del concepto de Educación Superior. Alternativas en Psicología. Alternativas en Psicología, 16(27), 34-41.

Hernandez, R.M. (2017). Impacto de las TIC en la educación: Retos y Perspectivas. Propósitos y Representaciones, 5(1), 325-347. Doi: http:// dx.doi.org/10.20511/pyr2017.v5n1.149

Hollander, E.P. (2000). Principios y métodos de psicología social. Buenos Aires-Nueva York, Oxford UniversitPress.

Irigoyen, J., Jiménez, M., \& Acuña, C. (2011).Competencias y educación superior. Revista Mexicana de Investigación Educativa, 16(48), 243-266.

Izquierdo, JM., \& Pardo, ML. (2007). Las Tecnologías de la Información y las Comunicaciones (TIC) en la gestión académica del proceso docente educativo en la educación superior. Revista Pedagógica Universitaria 12(1), 58-68.

Jacovkis, P. M. (2012). Las TIC en América Latina: historia e impacto social. Revista Iberoamericana de Ciencia, Tecnología y Sociedad, 6(18), 63-64.

Kay R.H., \& Knaack L. (2009) Assessing learning, quality and engagement in learning objects: the Learning Object Evaluation Scale for Students (LOES-S). Educational Technology Research and Development, 57, 147-168. Doi: http://dx.doi.org/10.1007/s11423-008-9094-5

Llorente, M. C., Cabero, J. \& Barroso, J. (2015). El papel del profesorado y alumnado en los nuevos entornos tecnológicos. En J. Cabero \& J. Barroso (Eds.), Nuevos retos en tecnología educativa (pp. 217-236). Madrid: Síntesis.

Marín V., \& Romero López, M. (2009). La formación docente universitaria a través de las TICs. Pixel-Bit. Revista de Medios y Educación, 35, 97103. Recuperaqdo de: http://hdl.handle.net/11441/22601 
Marqués, G. (2004). Metodología Didáctica y TIC en la enseñanza universitaria. Recuperado de: http://dewey.uab.es/PMARQUES/caceres.htm

Ortíz, A.M., Almanzán, L., Peñaherrera, M., \&Cachón, J. (2014). Formación en TIC de futuros maestros desde el análisis de la práctica en la Universidad de Jaén. Pixel-Bit. Revista de Medios y Educación, 44, 127142 Doi: https://doi.org/10.12795/pixelbit.2014.i44.09

Rangel, P., \& Peñalosa, E. (2013). Alfabetización digital en docentes de educación superior: construcción y prueba empírica de un instrumento de evaluación. Pixel-Bit. Revista de Medios y Educación, 43, 9-23. Doi: https://doi.org/10.12795/pixelbit.2013.i43.01

Rosario, H., \& Vázquez, L. (2012). Formación del docente universitario en el uso de TIC. Caso de las universidades públicas y privadas. Pixel-Bit. Revista de Medios y Educación, 41, 163-171. Recuperado de: http://hdl. handle.net/11441/22653

Salinas, J. (2004). Innovación docente y uso de las TIC en la enseñanza universitaria. Revista Universidad y Sociedad del Conocimiento, 1(1), 1-16.

Schubert, V., Medina J., \& do Prado, M. (2011) Proceso de construcción del conocimiento pedagógico del docente universitario de enfermería. Revista Latinoamericana Enfermagem, 19(2), 1-8.

Touriñan, J. M. (2005). Teoría de la Educación: Desarrollo administrativo de la cátedra y pertenencia del área. Revista Española de Pedagogía, 180, 265-280.

Twining, P. (2002). Conceptualising computer use in education: introducing the Computer Practice Framework (CPF). British Educational Research Journal,28(1),95-110.Doi:https://doi.org/10.1080/01411920120109775

Unesco (09 de octubre, 1998). Declaración mundial sobre la educación superior en el siglo XXI: Visión y acción. Recuperado de: http://www. unesco.org/education/educprog/wche/declaration_spa.htm 